\title{
The Askaryan Radio Array
}

\author{
Kara D. Hoffman
}

\author{
Physics Department, University of Maryland, \\ College, Park, MD 20742 U.S.A. \\ email: kara@umd.edu
}

\begin{abstract}
Ultra high energy cosmogenic neutrinos could be most efficiently detected in dense, radio frequency $(\mathrm{RF})$ transparent media via the Askaryan effect. Building on the expertise gained by RICE, ANITA and IceCube's radio extension in the use of the Askaryan effect in cold Antarctic ice, we are currently developing an antenna array known as ARA (The Askaryan Radio Array) to be installed in boreholes extending $200 \mathrm{~m}$ below the surface of the ice near the geographic South Pole. The unprecedented scale of ARA, which will cover a fiducial area of $\approx 100$ square kilometers, was chosen to ensure the detection of the flux of neutrinos suggested by the observation of a drop in high energy cosmic ray flux consistent with the GZK cutoff by HiRes and the Pierre Auger Observatory. Funding to develop the instrumentation and install the first prototypes has been granted, and the first components of ARA were installed during the austral summer of 2010-2011. Within 3 years of commencing operation, the full ARA will exceed the sensitivity of any other instrument in the 0.1-10 EeV energy range by an order of magnitude. The primary goal of the ARA array is to establish the absolute cosmogenic neutrino flux through a modest number of events. This information would frame the performance requirements needed to expand the array in the future to measure a larger number of neutrinos with greater angular precision in order to study their spectrum and origins.
\end{abstract}

Keywords. neutrinos, diffuse radiation

\section{Introduction}

One of the most tantalizing questions in astronomy and astrophysics, namely the origin and the evolution of the cosmic accelerators that produce the highest energy (UHE) cosmic rays, may be best addressed through the observation of UHE cosmogenic neutrinos. Astrophysical proton acceleration sites must also be neutrino emitters, since protons must undergo pion photo production in order to escape the magnetic field of their source (Ahlers et al., 2011). The decay chain of the daughter pions will contain gamma rays and neutrinos. Unlike the parent proton, neutrinos travel from their source undeflected by magnetic fields and largely unimpeded by interactions with intervening material. At high energies (above $10^{16} \mathrm{eV}$ ), neutrinos could be most efficiently detected in dense, radio frequency (RF) transparent media via the Askaryan (Askaryan, 1962) effect. The abundant cold ice covering the geographic South Pole, with its exceptional RF clarity, has been host to several pioneering efforts to develop this approach, including RICE (Kravchenko et al., 2003) and ANITA (Gorham, 2006).

Building on the expertise gained in these efforts, and the infrastructure developed in the construction of the IceCube optical Cherenkov observatory, we are currently installing prototype instrumentation for an array, known as ARA (The Askaryan Radio Array), in the deep ice near the geographic South Pole. Above $\approx 10^{15} \mathrm{eV}$ the power emitted in RF excedes that in optical, and the attenuation length of RF in cold ice is long - on the order of a kilometer - allowing coverage with a larger, sparse array at low cost. South Polar ice is perhaps the most extensively studied on Earth. The combination of ice thickness and 
favorable radiofrequency dielectric characteristics (see Figure 3), as well as the excellent scientific infrastructure, makes the site unparalleled for the location of this array.

With an instrumented area of an unprecedented $\approx 100 \mathrm{~km}^{2}$, ARA's size was chosen to ensure the detection of the flux of neutrinos implied by the observation of a steep drop in the cosmic ray spectrum at $\approx 10^{19.5} \mathrm{eV}$ (Greisen, 1966, Zatsepin \& Kuzmin, 1966) by HiRes (Abbasi et al., 2008) and the Pierre Auger Observatory (Abraham et al., 2008). Greisen, Zatsepin and Kuzmin (GZK) predicted the existence of this cutoff, which they attribute to the scattering of cosmic ray protons on the cosmic microwave background (CMB) photons via:

$$
\begin{aligned}
& p+\gamma_{2.7 K} \rightarrow \Delta^{+} \rightarrow n+\quad \pi^{+} \\
& \hookrightarrow \quad \mu \nu_{\mu} \\
& \hookrightarrow e \nu_{e} \nu_{\mu} .
\end{aligned}
$$

The presence of neutrinos in the decay of the resulting $\Delta$ resonance and the fact that ultra-high energy cosmic rays (UHECR) have been observed, and almost certainly include a significant proton fraction, implies the existence of neutrinos at $10^{17}-10^{19} \mathrm{eV}$, unless, coincidentally, this energy corresponds to a limit in the energy that can be attained by cosmogenic accelerators. The observation of ultra high energy cosmogenic neutrinos is needed to confirm that the observed dip is the result of the GZK process. The spectrum of astrophysical neutrinos is expected to follow the spectrum of cosmic rays, whose rates fall exponentially as a function of energy. However, if the drop in flux is attributable to the GZK process, the precise magnitude of the resultant neutrino flux will depend on the proton/iron composition of the cosmic rays, as well as the distribution of their sources in space. Thus, a measurement of ultra high energy neutrinos would also give an indirect measurement of the cosmic ray species.

Within 3 years of commencing operation, the full ARA will exceed the sensitivity of any other neutrino observatory in the 0.1-10 EeV energy range by an order of magnitude. Because the antennas will be deployed in boreholes extending below the firn layer to 200 $m$ depth, it will have the ability to distinguish surface noise from sources originating in the ice cap, otherwise not possible in the ballon borne approach employed by ANITA (ANITAinstr). Even under the extreme assumption that UHE cosmic rays are pure iron, ARA will have sufficient sensitivity to establish the presence or absence of the secondary UHE neutrinos produced in the GZK interaction. Such an observatory would also provide an unique probe of long baseline high energy neutrino interactions unattainable with any man-made neutrino beam.

\section{Array Baseline Design}

The baseline design of ARA consists of 37 antenna clusters or "stations" arranged $2 \mathrm{~km}$ apart on a triangular grid, as shown in Figure 1. The primary goal of the ARA array is to establish the absolute cosmogenic neutrino flux through a modest number of gold plated events. We have therefore adopted a geometry in which a single localized cluster may act as a standalone array, which trades precise angular resolution for increased coverage. Because each local cluster has the ability self-trigger, this also avoids the complications of long trigger windows and deep buffers that would otherwise arise in triggering a sparse, large scale array.

The configuration of the ARA clusters is shown in Figure 2. Each cluster will consist of four strings of receiver antennas and associated amplifiers installed in boreholes of 


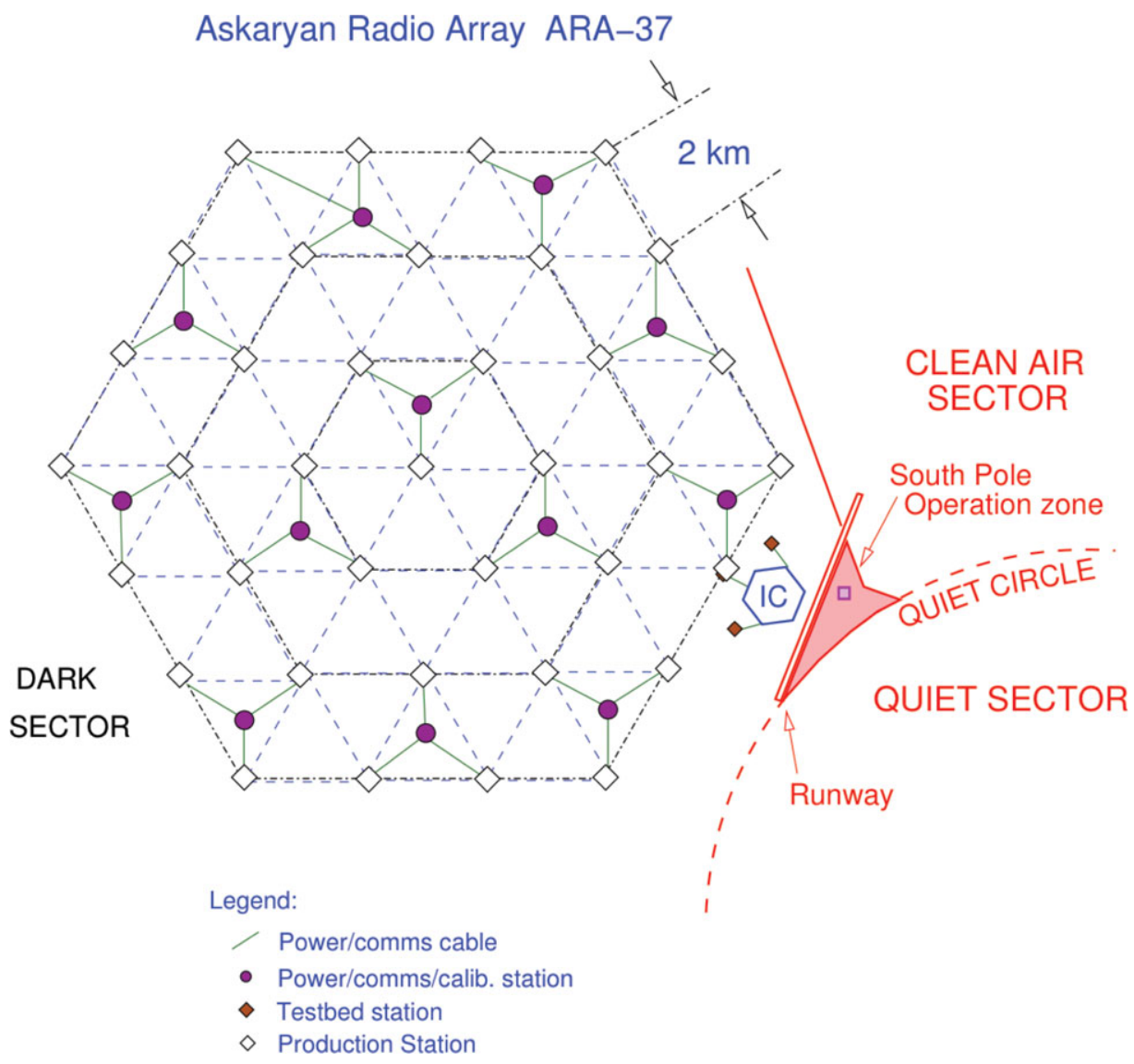

Figure 1. Planned layout of the 37 ARA stations with respect to the South Pole Station and associated sectors. The footprint of IceCube, which covers a square kilometer, is shown by the blue hexagon. The geographic South Pole is marked by the magenta square in the South Pole operation zone.

approximately 6 inches $(15 \mathrm{~cm})$ in diameter and extending $200 \mathrm{~m}$ deep into the icecap. The depth was chosen to allow the instrumentation to be placed below the first $100 \mathrm{~m}$ of the icecap, known as the "firn" layer, which has a varying index of refraction due to the gradual compactification of snow into ice. The firn layer would reflect away much of the power and complicate the ray tracing used in event reconstruction. The drilling technology and fuel constraints limit the depth and diameter of the borehole.

Each string will contain 2 pairs of antennas, each pair consisting of a horizontally polarized antenna and a vertically polarized antenna. The antenna pairs will be separated by a distance of $10-50 \mathrm{~m}$ for depth discrimination. By combining information from the two antenna polarizations, the entire Cherenkov cone may be reconstructed in the data analysis, allowing for more precise vertex reconstruction and additional background rejection. Each antenna will be coupled to a low noise amplifier with low and high pass filters that select the sensitive frequency range, which extends from $100 \mathrm{MHz}$ to $900 \mathrm{MHz}$. The digitization electronics is a descendent of those developed for ANITA (Gorham et al., 2009, Varner et al., 2007). The cluster of four strings, along with the surface trigger logic, and two additional antennas to transmit calibration pulses, form a station. 


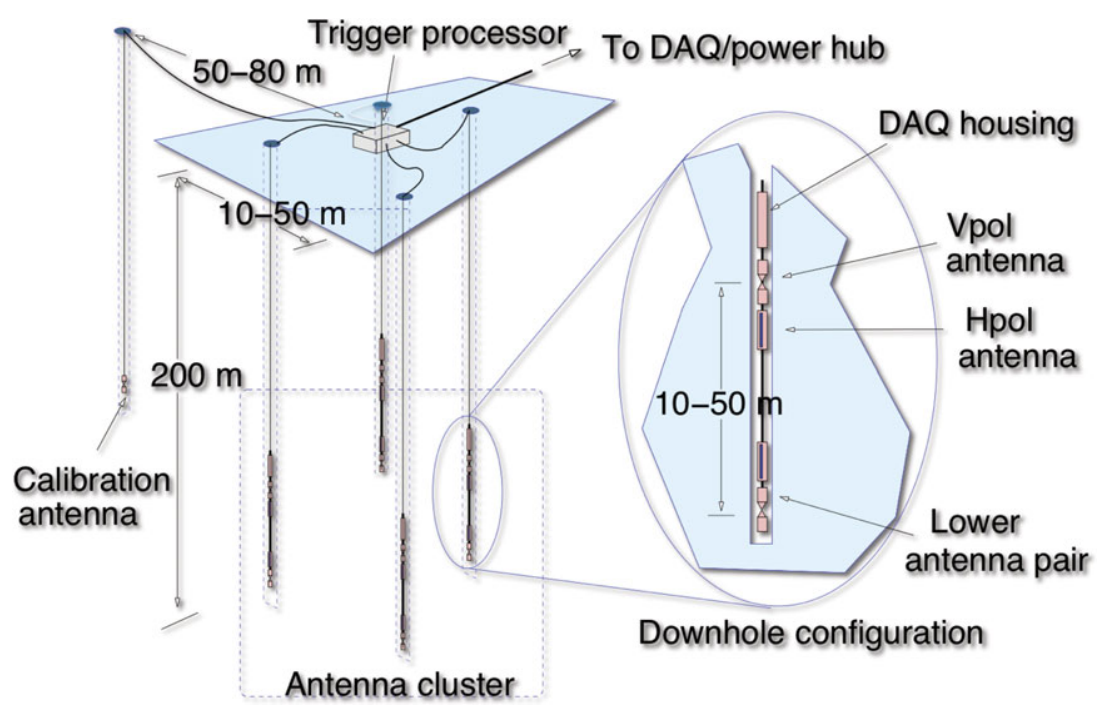

Figure 2. ARA Station layout and antenna cluster geometry.

\section{Prototype Construction and Performance}

One of the goals of the first season of ARA construction was to test prototypes of the hardware. This prototype hardware can, in turn, be used to probe the noise environment at a distance of one kilometer from the IceCube array. Toward this goal, a "test bed" of shallow antennas was installed during the austral summer of 2010-2011. It consists of pairs of antennas, a "batwing" for horizontal polarization and a discone for vertical polarization, installed in trenches $3 \mathrm{~m}$ below the surface of the snow, as well as two low frequency antennas installed near the surface. An additional four pairs of antennas were installed in 6 inch boreholes drilled $40 \mathrm{~m}$ into the ice. The design of the antenna pairs for the narrow boreholes, which served as prototypes for the ARA stations, were constrained by the size of the borehole, and the requirement that the power and communications cable has to pass through the center of the antenna. The deeper $40 \mathrm{~m}$ boreholes also served to test different drilling technologies. Because we intend to build a sparse, large scale array, any drill used must be portable, and must be capable of drilling to the desired depth in a matter of hours.

Another goal of the first season of ARA was to measure the RF properties of ice to a sufficient degree to understand the performance of the array. The RF attenuation length decreases as a function of temperature, and the temperature of the ice at the South Pole has been precisely surveyed and has been found to vary from $-50^{\circ} \mathrm{C}$ at the surface to $-10^{\circ} \mathrm{C}$ at $2,800 \mathrm{~m}$ depth (Price et al., 2002). In addition, the refractive index varies as a function of density, resulting in a lensing effect near near the top of the polar ice cap, where the snow is gradually compacting into ice. The RF propagation could also conceivably be effected by layering in the ice that could act as a waveguide. This "birefringence", if it is present, could also complicate the analysis of data. To investigate the RF properties of the ice, the ARA collaboration took advantage of the drilling of deep holes for the installation of the final IceCube strings to parasitically install three $5 \mathrm{kV}$ broadband pulser and transmitter antenna assemblies at depths of 1,400 and 2,500 meters. The antenna was designed to wrap around the IceCube cable to eliminate uncertainty in the transmission pattern from cable shadowing effects. The siting of these transmitters will allow the RF properties of the deep ice to be probed without the 


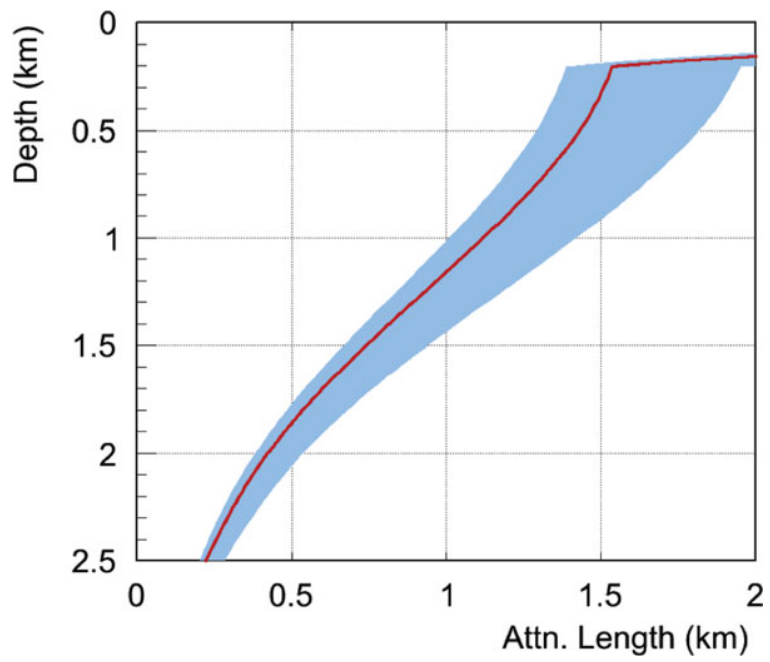

Figure 3. Ice Attenuation measurements made at the South Pole in 2011 measured from direct transmission from a source $2,500 \mathrm{~m}$ in depth along a $3.2 \mathrm{~km}$ slant depth.

uncertainties associated with the bottom reflection, and the high voltage of these pulsers will allow them to be detected from many attenuation lengths away - well beyond the extent of IceCube, at the testbed, and within range of a large fraction of the planned ARA stations. The deepest transmitter is located at a slant depth of $3.2 \mathrm{~km}$ from the testbed, allowing a direct measurement of ice attenuation length over an unprecedented distance. The resulting measurement is shown in Figure 3. This will serve as a valuable calibration tool for ARA.

The testbed and high voltage transmitters have been operating for nearly two years, and the full results have been published (Allison et al., 2012). The noise environment has been found to be very favorable, with the spectral noise density plotted for the low frequency surface antennas shown in Figure 4. The measurement agrees with the ambient thermal noise above $150 \mathrm{MHz}$. Below $150 \mathrm{MHz}$, it is dominated by the galactic thermal noise. A modulation of the galactic noise is seen as the horizontally oriented antenna aligns and anti-aligns with the plane of the galaxy, which is inclined at $\approx 63^{\circ}$ with respect to the South Pole, over a period of a sidereal day. Sources of manmade interference include weather ballon launches that employ a $400 \mathrm{MHz}$ transponder for data telemetry, and a $129.3 \mathrm{MHz}$ communication channel used for incoming and departing aircraft. The amount of interference decreases significantly over the winter. Overall, we expect to loose $3 \%$ lifetime due to interference.

Local calibration pulsers installed within the testbed have been used to determine the geometry of the array in situ. By cross correlating the transmitted reference signal in the Vpol antennas, the timing of the transmissions for an ensemble of pulses can be measured to $4.5 \mathrm{ps}$, with a standard deviation of $136 \mathrm{ps}$. This corresponds to a $1 \mathrm{~mm}$ error in the position of the antennas.

In the austral summer following the installation of the testbed, one full prototype ARA station was installed. It closely resembles the ARA stations as proposed, except that boreholes of only $100 \mathrm{~m}$ were achieved due to the ongoing development of the drill. An additional two prototypes are planned for installation during the austral summer of 2012-2013 which include upgrades to the data-acquisition system, which will be near the final design. 


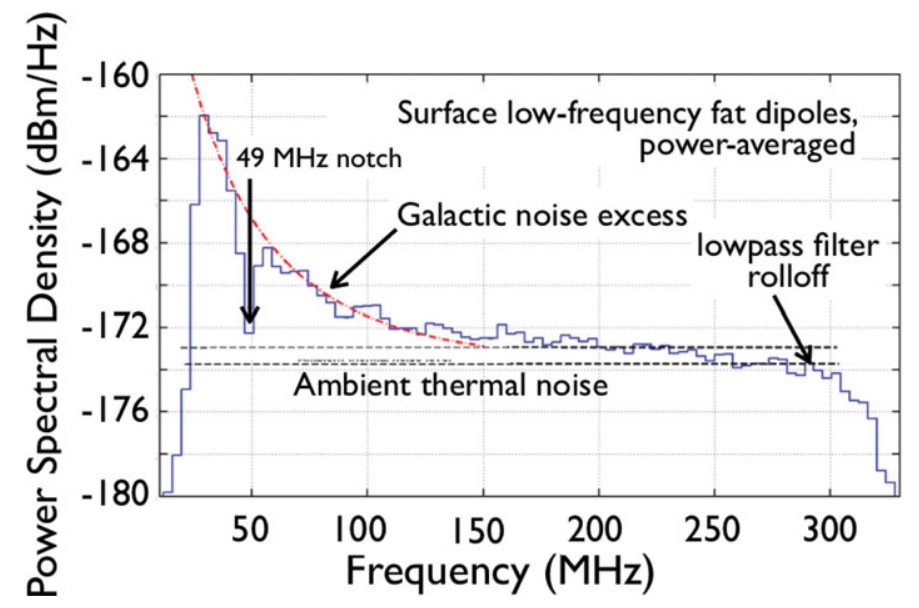

Figure 4. Spectrum of the noise density for surface dipoles measured at the ARA testbed.

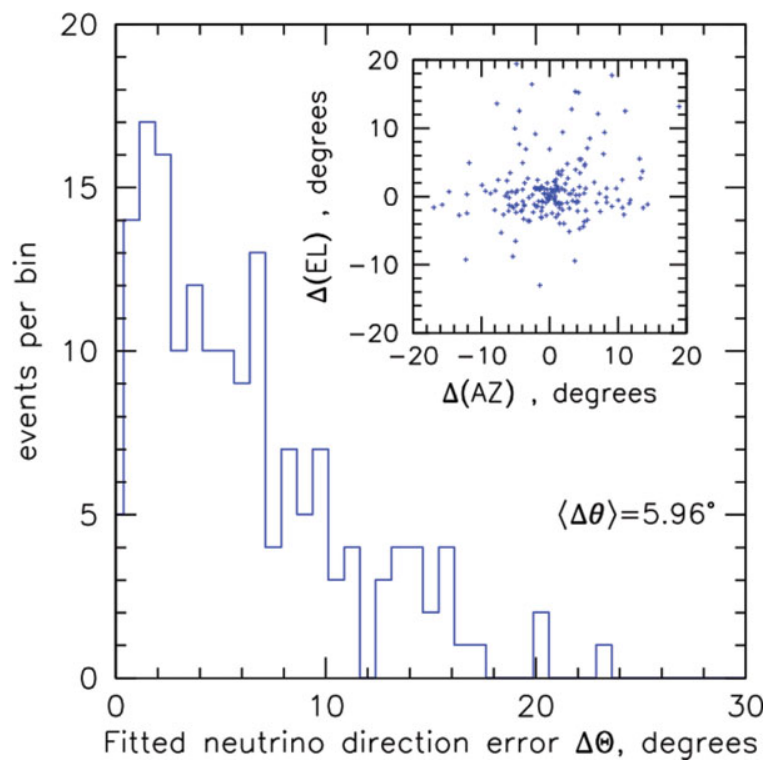

Figure 5. Distribution of fractional range errors in single-antenna-cluster reconstruction of neutrino interaction vertex. Distribution of polar angle errors for full reconstruction of the incoming neutrino direction, using vertex reconstruction, amplitude and polarization information. Inset: the 2-D distribution of reconstructed directions relative to true neutrino direction.

\section{Performance of the Proposed Array}

\subsection{Angular resolution}

Because the goal of the ARA array is to make the first detections of neutrinos via the Askaryan effect, and establish the neutrino flux, angular resolution was not emphasized in the design. However, good up-down discrimination is essential in vetoing surface anthropogenic noise. The simulated angular resolution of the ARA baseline array is shown in Figure 5, with a median angular resolution of $5.96^{\circ}$. This resolution assumes a single station detection, and is dominated by the azimuthal error. The error in the zenith angle reconstruction is about one degree. 


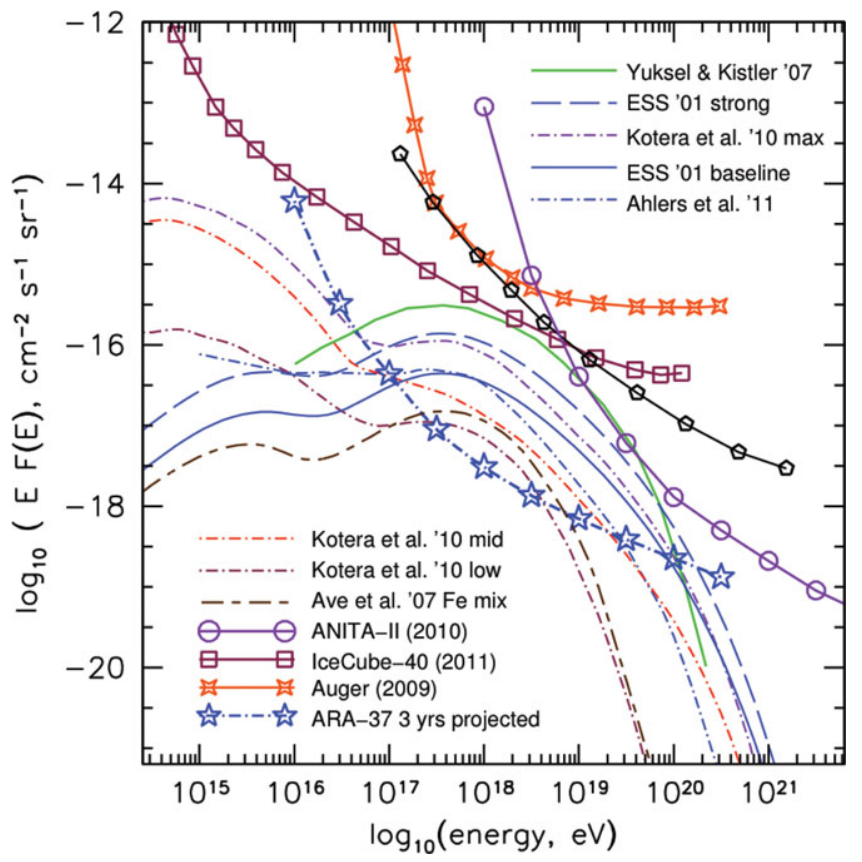

Figure 6. Compilation of sensitivity estimates from existing instruments, published limits, and a range of GZK neutrino models, along with the expected 3 year ARA sensitivity.

\subsection{Sensitivity to the neutrino flux}

The limit on the cosmogenic neutrino flux from ARA after 3 years of operation of the full 37 station array is shown by the blue stars in Figure 6. Also shown are the expected flux upper limits from several competing experiments including RICE (Kravchenko et al., 2006, pentagons), ANITA (Gorham, 2010, circles), Auger (Abraham et al., 2009, stars) and IceCube (Abbasi et al., 2011, squares). The reason that the sensitive energy range of ARA was chosen to be lower than ANITA's was to optimize the instrument so that its peak sensitivity lies in the region where the cosmogenic neutrino flux is greatest, thereby maximizing the probability of detection. Also shown are several theoretical flux calculations based on different assumptions about the cosmic ray composition and the source evolution, including strong source evolution models (Engel et al., 2001, Yuksel \& Kistler, 2007), mixed iron composition models (Kotera et al., 2010, Ave et al., 2005), and models incorporating constraints from the Fermi diffuse gamma ray flux (Ahlers et al., 2010). ARA has the greatest sensitivity of any neutrino telescope in the energy interval from $10^{17}-10^{19.5} \mathrm{eV}$. ARA can either detect or exclude a cosmogenic neutrino flux even in the more pessimistic scenario of an all iron cosmic ray spectrum (Ave et al., 2005). ARA is intended as a discovery instrument.

The prototyping phase for ARA is nearly complete. After funding to build the balance is secured, the array could be completed in five years. Once the flux of cosmogenic neutrinos is measured, this could inform the design of an observatory class array with precise angular resolution to study the origin and evolution of the highest energy cosmic ray accelerators. 


\section{Acknowledgements}

We thank the National Science Foundation for their generous support through Grant NSF ANT-1002483. We are very grateful to Raytheon Polar Services Corporation and the Lockheed Martin Antarctic Support Division for their excellent field support at Amundsen-Scott Station, and the IceCube drillers and specialists who helped with our installation.

\section{References}

Abbasi, R. et al. (2008). Phys. Rev. Lett., 100, 101101.

Abbasi, R. et al. (2011). Phys. Rev., D83, 093003.

Abraham, J. et al. (2008). Phys. Rev. Lett., 101, 061101.

Abraham, J. et al. (2009). Phys. Rev., D79, 102001.

Ahlers, M., Anchordoqui, L., Gonzalez-Garcia, M., Halzen, F. \& Sarkar, S. (2010). Astropart. Phys., 34, 106-115.

Ahlers, M., Gonzalez-Garcia, M. \& Halzen, F. (2011). Astropart. Phys., 35, 87-94.

Allison, P., Auffenberg, J., Bard, R., Beatty, J., Besson, D. et al. (2012). Astropart. Phys., 35, $457-477$.

Askaryan, G. A. (1962). JETP Lett., 14, 441.

Ave, M., Busca, N., Olinto, A. V., Watson, A. A. \& Yamamoto, T. (2005). Astropart. Phys., 23, 19-29.

Engel, R., Seckel, D. \& Stanev, T. (2001). Phys. Rev., D64, 093010.

Gorham, P. W. et al. (2009). Astropart. Phys., 32, 10-41.

Gorham, P. W. (2006). Int. J. Mod. Phys., A21S1, 158-162.

Gorham, P. W. (2010). Phys. Rev., D82, 022004.

Greisen, K. (1966). Phys. Rev. Lett., 16, 748-750.

Kotera, K., Allard, D. \& Olinto, A. (2010). JCAP, 1010, 013.

Kravchenko, I. et al. (2003). Astropart. Phys., 19, 15-36.

Kravchenko, I. et al. (2006). Phys. Rev., D73, 082002.

Price, P. B., et al. (2002). Proc. Nat. Acad. Sciences USA, 99, 7844-7847.

Varner, G. S., Ruckman, L. L., Gorham, P. W., Nam, J. W., Nichol, R. J., Cao, J. \& Wilcox, M. (2007). Nucl. Instrum. Meth., A583, 447-460.

Yuksel, H. \& Kistler, M. D. (2007). Phys. Rev., D75, 083004.

Zatsepin, G. T. \& Kuzmin, V. A. (1966). JETP Lett., 4, 78-80. 